\title{
Topological Nature of the Phonon Hall Effect
}

\author{
Lifa Zhang,, , * Jie Ren,, 2, , \\ ${ }^{1}$ Department of Physics and Centre for Computational Science and Engineering, \\ National University of Singapore, Singapore 117542, Republic of Singapore \\ ${ }^{2}$ NUS Graduate School for Integrative Sciences and Engineering, Singapore 117456, Republic of Singapore
}

(Dated: 02 Aug 2010, Revised 25 Nov 2010)

\begin{abstract}
We provide a topological understanding of the phonon Hall effect in dielectrics with Raman spinphonon coupling. A general expression for phonon Hall conductivity is obtained in terms of the Berry curvature of band structures. We find a nonmonotonic behavior of phonon Hall conductivity as a function of the magnetic field. Moreover, we observe a phase transition in the phonon Hall effect, which corresponds to the sudden change of band topology, characterized by the altering of integer Chern numbers. This can be explained by touching and splitting of phonon bands.

PACS numbers: 66.70.-f, 72.10.Bg, 03.65.Vf, 72.15.Gd
\end{abstract}

Recent years have witnessed a rapid development of an emerging field - phononics, the science and technology of controlling heat flow and processing information with phonons [1]. Indeed, in parallel with electronics, various functional thermal devices such as thermal diode [2], thermal transistor [3], thermal logic gates [4] and thermal memory [5], etc., have been proposed to manipulate and control phonons, the carrier of heat energy and information. However, different from electrons, phonons as neutral quasiparticles, cannot directly couple to the magnetic field through the Lorentz force. Therefore, it is a surprise that Strohm, Rikken, and Wyder observed the phonon Hall effect (PHE) - the appearance of a temperature difference in the direction perpendicular to both the applied magnetic field and the heat current flowing through an ionic paramagnetic dielectric sample [6]. It was confirmed later by Inyushkin and Taldenkov [7]. Since then, several theoretical explanations have been proposed [2-4] to understand this novel phenomenon.

For electronic transport properties in various quantum, spin, or anomalous Hall effects [11 13], topological Berry phase has been successfully used to understand the underlying mechanism [14]. Such an elegant connection between mathematics and physics provides a broad and deep understanding of basic material properties. However, because of the very different nature of electrons and phonons, a topological picture related to the PHE is not straightforward and obvious, and therefore, is still lacking.

In this Letter, we explore the topology of phonon bands in a two-dimensional honeycomb lattice with Raman type spin-phonon interaction. A general expression for phonon Hall conductivity in terms of Berry curvature is derived. The phonon Hall effect is not quantized, although the Chern numbers are quantized to integers. We find that there exists a phase transition associated with the PHE, due to the discontinuous jump of Chern numbers.

We start with a Hamiltonian for an ionic crystal lattice in a uniform external magnetic field [1], which reads in a compact form as

$$
\begin{aligned}
H & =\frac{1}{2}(p-\tilde{A} u)^{T}(p-\tilde{A} u)+\frac{1}{2} u^{T} K u \\
& =\frac{1}{2} p^{T} p+\frac{1}{2} u^{T}\left(K-\tilde{A}^{2}\right) u+u^{T} \tilde{A} p .
\end{aligned}
$$

Here, $u$ is a column vector of displacements from lattice equilibrium positions for all the degrees of freedom, multiplied by the square root of mass, $p$ is the conjugate momentum vector, and $K$ is the force constant matrix. The superscript $T$ stands for the matrix transpose. $\tilde{A}$ is an antisymmetric real matrix, which is block diagonal with elements $\Lambda=\left(\begin{array}{rr}0 & h \\ -h & 0\end{array}\right)$ (in two dimensions), where $h$ is proportional to the magnitude of the applied magnetic field, and has the dimension of frequency. For simplicity, we will call $h$ the magnetic field later. The on-site term, $u^{T} \tilde{A} p$, can be interpreted as the Raman (or spin-phonon) interaction [16]. The Hamiltonian (1) is positive definite.

By applying Bloch's theorem, we can describe the system by the polarization vector $x=(\mu, \epsilon)^{T}$, where $\mu$ and $\epsilon$ are associated with the momenta and coordinates, respectively. The equation of motion can be expressed as

$$
i \frac{\partial}{\partial t} x=H_{\mathrm{eff}} x, \quad H_{\mathrm{eff}}=i\left(\begin{array}{cc}
-A & -D \\
I & -A
\end{array}\right),
$$

where $D(\mathbf{k})=-A^{2}+\sum_{l^{\prime}} K_{l, l^{\prime}} e^{i\left(\mathbf{R}_{l^{\prime}}-\mathbf{R}_{l}\right) \cdot \mathbf{k}}$ is the dynamic matrix as a function of wave vector $\mathbf{k} ; K_{l, l^{\prime}}$ is the submatrix between unit cell $l$ and $l^{\prime}$ in the full spring constant matrix $K ; \mathbf{R}_{l}$ is the real-space lattice vector; $A$ is block diagonal with elements $\Lambda$, and $I$ is an identity matrix. Here, $D, A, K_{l, l^{\prime}}$, and $I$ are all $4 \times 4$ matrices for the twodimensional honeycomb lattice. The eigenvalue problem of the equation of motion (2) reads:

$$
H_{\mathrm{eff}} x_{\sigma}=\omega_{\sigma} x_{\sigma},
$$

where $x_{\sigma}=\left(\mu_{\sigma}, \epsilon_{\sigma}\right)^{T}$ is the right eigenvector of the $\sigma$ th branch and $\omega_{\sigma}$ is the corresponding eigenfrequency. Because of the non-Hermitian nature of $H_{\mathrm{eff}}$, the left 
eigenvector is different, and is given by $\tilde{x}_{\sigma}^{T}=\left(\tilde{\mu}_{\sigma}, \tilde{\epsilon}_{\sigma}\right)=$ $\left(\epsilon_{\sigma}^{\dagger},-\mu_{\sigma}^{\dagger}\right) /\left(-2 i \omega_{\sigma}\right)$. The orthonormal condition is $\epsilon_{\sigma}^{\dagger} \epsilon_{\sigma^{\prime}}+$ $\frac{i}{\omega_{\sigma}} \epsilon_{\sigma}^{\dagger} A \epsilon_{\sigma^{\prime}}=\delta_{\sigma, \sigma^{\prime}}[\underline{4}]$.

By taking into account only positive eigenfrequency modes, displacement and momentum operators can be written in the second quantization form. From the definition of energy current density $\mathbf{J}=\frac{1}{2 V} \sum_{l, l^{\prime}}\left(\mathbf{R}_{l}-\right.$ $\left.\mathbf{R}_{l^{\prime}}\right) u_{l}^{T} K_{l, l^{\prime}} \dot{u}_{l^{\prime}}$ 2, 3, 3], the current density vector can be expressed as

$$
\mathbf{J}=\mathbf{J}_{\mathbf{1}}\left(a^{\dagger} a\right)+\mathbf{J}_{\mathbf{2}}\left(a^{\dagger} a^{\dagger}, a a\right) .
$$

Here, $\mathbf{J}_{\mathbf{1}}=\frac{\hbar}{4 V} \sum_{k, k^{\prime}} \frac{\omega_{k}+\omega_{k^{\prime}}}{\sqrt{\omega_{k} \omega_{k^{\prime}}}} \epsilon_{k}^{\dagger} \frac{\partial D(\mathbf{k})}{\partial \mathbf{k}} \epsilon_{k^{\prime}} a_{k}^{\dagger} a_{k^{\prime}} e^{i\left(\omega_{k}-\omega_{k^{\prime}}\right) t} \delta_{\mathbf{k}, \mathbf{k}^{\prime}}$, and $\mathbf{J}_{\mathbf{2}}=\frac{\hbar}{4 V} \sum_{k, k^{\prime}} \sqrt{\frac{\omega_{k^{\prime}}}{\omega_{k}}}\left(\epsilon_{k}^{\dagger} \frac{\partial D(\mathbf{k})}{\partial \mathbf{k}} \epsilon_{k^{\prime}}^{*} a_{k}^{\dagger} a_{k^{\prime}}^{\dagger} e^{i\left(\omega_{k}+\omega_{k^{\prime}}\right) t}+\right.$ $\left.\epsilon_{k}^{T} \frac{\partial D^{*}(\mathbf{k})}{\partial \mathbf{k}} \epsilon_{k^{\prime}} a_{k} a_{k^{\prime}} e^{-i\left(\omega_{k}+\omega_{k^{\prime}}\right) t}\right) \delta_{\mathbf{k},-\mathbf{k}^{\prime}}$, where $k=(\mathbf{k}, \sigma)$ considers both the wavevector and the phonon branch. It should be noted that the $a^{\dagger} a^{\dagger}$ and $a a$ terms also contribute to the off-diagonal elements of thermal conductivity tensor, although they have no contribution to the average heat flux. The diagonal term $\epsilon_{k}^{\dagger} \frac{\partial D(\mathbf{k})}{\partial \mathbf{k}} \epsilon_{k}$ in $\mathbf{J}_{\mathbf{1}}$ corresponds to $\omega_{\sigma} \frac{\partial \omega_{\sigma}}{\partial \mathbf{k}}$. Only the off-diagonal terms in $\mathbf{J}_{\mathbf{1}}$ and $\mathbf{J}_{\mathbf{2}}$ contribute to the Hall conductivity, which can be regarded as the contribution from anomalous velocities similar to the one in the intrinsic anomalous Hall effect [12]. Using the Green-Kubo formula $\kappa_{x y}=\frac{V}{\hbar T} \int_{0}^{\beta \hbar} d \lambda \int_{0}^{\infty} d t\left\langle J^{x}(-i \lambda) J^{y}(t)\right\rangle_{\text {eq }}[10]$, one can obtain phonon Hall conductivity as [16]:

$$
\begin{aligned}
\kappa_{x y}= & \frac{\hbar}{8 V T} \sum_{\sigma \neq \sigma^{\prime}} f\left(\omega_{\sigma}\right)\left(\omega_{\sigma}+\omega_{\sigma^{\prime}}\right)^{2} \times \\
& \frac{i}{4 \omega_{\sigma} \omega_{\sigma^{\prime}}} \frac{\epsilon_{\sigma}^{\dagger} \frac{\partial D}{\partial k_{x}} \epsilon_{\sigma^{\prime}} \epsilon_{\sigma^{\prime}}^{\dagger} \frac{\partial D}{\partial k_{y}} \epsilon_{\sigma}-\left(k_{x} \leftrightarrow k_{y}\right)}{\left(\omega_{\sigma}-\omega_{\sigma^{\prime}}\right)^{2}},
\end{aligned}
$$

where $f\left(\omega_{\sigma}\right)=\left(e^{\hbar \omega_{\sigma} /\left(k_{B} T\right)}-1\right)^{-1}$ is the Bose distribution function, $V$ is the total volume of the sample, and the phonon branch index $\sigma$ here includes both the positive and negative eigenvalues without restrictions. It can be proved that the phonon Hall conductivity $\kappa_{x y}$ satisfies the Onsager reciprocal relations [16].

In Fig. 1 we show the phonon Hall conductivity of honeycomb lattices calculated from Eq. (5). The parameters used in our numerical calculations are the same as in Ref. [4]. The coupling matrix between two sites is configured such that the longitudinal spring constant is $K_{L}=0.144 \mathrm{eV} /\left(\mathrm{u} \AA^{2}\right)$ and the transverse one $K_{T}$ is 4 times smaller. The unit cell lattice vectors are $(a, 0)$ and $(a / 2, a \sqrt{3} / 2)$ with $a=1 \AA$.

It is found that when $h$ is small, $\kappa_{x y}$ is proportional to $h$ [16], while the dependence becomes nonlinear when $h$ is large. As $h$ is further increased, $\kappa_{x y}$ increases before it reaches a maximum at certain value of $h$. Then $\kappa_{x y}$ decreases and goes to zero at very large $h$. This can be understood as follows: numerical calculation shows that
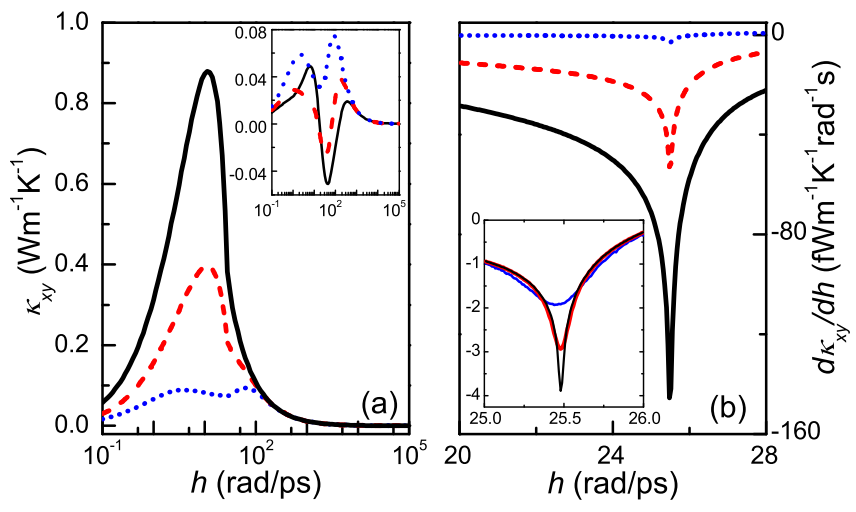

FIG. 1: (color online) (a) Phonon Hall conductivity vs magnetic field for different temperatures. The dotted, dashed, and solid lines correspond to $T=50,100$, and $300 \mathrm{~K}$, respectively. The inset shows $h$-dependence of $\kappa_{x y}$ at low temperatures: $T=10$ (solid line), 20 (dashed line), and $40 \mathrm{~K}$ (dotted line). (b) $d \kappa_{x y} / d h$ as a function of $h$ at different temperatures: $T=50$ (dotted line), 100 (dashed line), and $300 \mathrm{~K}$ (solid line); here $N_{L}=400$. The inset in (b) shows the $h$ dependence of $d \kappa_{x y} / d h$ for different size $N_{L}$ at $T=50 \mathrm{~K}$, around $h \approx 25.5 \mathrm{rad} / \mathrm{ps}$; from top to bottom, $N_{L}=80,320$, and 1280 , respectively.

$\omega_{\sigma} \approx \alpha h$, which can also be obtained from the equation $\left[\left(-i \omega_{\sigma}+A\right)^{2}+D\right] \epsilon_{\sigma}=0[16]$, thus we can obtain approximately $\kappa_{x y} \sim h^{2} /\left(e^{\beta \hbar \alpha h}-1\right)$ from Eq. (5). In the weak magnetic field limit $\kappa_{x y} \propto h$, while in the strong field limit, $\kappa_{x y} \rightarrow 0$. The on-site term $\tilde{A}^{2}$ in the Hamiltonian (11) increases with $h$ quadratically so as to blockade the phonon transport, which competes with the spin-phonon interaction. Therefore, as $h$ increases, $\kappa_{x y}$ first increases, then decreases and tends to zero at last. At low temperatures, $\kappa_{x y}$ oscillates around zero with the variation of $h$, as shown in the inset in Fig. 1(a).

There is a subtle singularity near $h \simeq 25 \mathrm{rad} / \mathrm{ps}$ in Fig. 1(a); we thus plot the first derivative of $\kappa_{x y}$ with respect to $h$ at different temperatures in Fig. 1(b). It shows that, at the relatively high temperatures, the first derivative of phonon Hall conductivity has a minimum at the magnetic field $h_{c} \simeq 25.4778 \mathrm{rad} / \mathrm{ps}$ for the finite-size sample $N_{L}=400$ (the sample has $N=N_{L}^{2}$ unit cells). The first derivative $d \kappa_{x y} / d h$ at the point $h_{c}$ diverges when the system size increases to infinity. The inset in Fig. 1(b) shows the finite-size effect. At the point $h_{c}$, the second derivative $d^{2} \kappa_{x y} / d h^{2}$ is discontinuous. Therefore, $h_{c}$ is a critical point for the PHE, across which a phase transition occurs. At low temperatures, the divergence of $d \kappa_{x y} / d h$ is not so evident as that at high temperatures. However, if the sample size becomes larger, the discontinuity of $d^{2} \kappa_{x y} / d h^{2}$ is more obvious, as illustrated in Fig. 1(b). For different temperatures, the phase transition occurs at exactly the same critical value $h_{c}$, which strongly suggests that the phase transition of the PHE is related to the topology of the phonon band structure. 
In the following, we would like to connect the PHE with the Berry phase to examine the underlying topological mechanism. As is wellknown, the band structure of crystals provides a natural platform to investigate the geometric phase effect. Since the wave-vector dependence of the polarization vectors is inherent to the Hall problems, the Berry phase effects are intuitively expected for the PHE in the momentum space. Following Berry's approach [14], we set $x(t)=e^{i \gamma_{\sigma}(t)-i \int_{0}^{t} d t^{\prime} \omega_{\sigma}\left(\mathbf{k}\left(t^{\prime}\right)\right)} x_{\sigma}(\mathbf{k}(t))$, and then insert it into Eq. (2). The Berry phase is obtained as $\gamma_{\sigma}=\oint \mathbf{A}_{\mathbf{k}}^{\sigma} \cdot d \mathbf{k}$, with $\mathbf{A}_{\mathbf{k}}^{\sigma}=i \tilde{x}_{\sigma}^{T} \frac{\partial x_{\sigma}}{\partial \mathbf{k}}$, and the Berry curvature emerges as

$$
\Omega_{k_{x} k_{y}}^{\sigma}=\frac{\partial}{\partial k_{x}} \mathbf{A}_{k_{y}}^{\sigma}-\frac{\partial}{\partial k_{y}} \mathbf{A}_{k_{x}}^{\sigma}=\sum_{\sigma^{\prime}, \sigma^{\prime} \neq \sigma} \Omega_{k_{x} k_{y}}^{\sigma \sigma^{\prime}}
$$

where,

$$
\Omega_{k_{x} k_{y}}^{\sigma \sigma^{\prime}}=\frac{i}{4 \omega_{\sigma} \omega_{\sigma^{\prime}}} \frac{\epsilon_{\sigma}^{\dagger} \frac{\partial D}{\partial k_{x}} \epsilon_{\sigma^{\prime}} \epsilon_{\sigma^{\prime}}^{\dagger} \frac{\partial D}{\partial k_{y}} \epsilon_{\sigma}-\epsilon_{\sigma}^{\dagger} \frac{\partial D}{\partial k_{y}} \epsilon_{\sigma^{\prime}} \epsilon_{\sigma^{\prime}}^{\dagger} \frac{\partial D}{\partial k_{x}} \epsilon_{\sigma}}{\left(\omega_{\sigma}-\omega_{\sigma^{\prime}}\right)^{2}}
$$

is the contribution to the Berry curvature of the band $\sigma$ from a different band $\sigma^{\prime}$. The associated topological Chern number is obtained through integrating the Berry curvature over the first Brillouin zone as

$$
C^{\sigma}=\frac{1}{2 \pi} \int_{\mathrm{BZ}} d k_{x} d k_{y} \Omega_{k_{x} k_{y}}^{\sigma}=\frac{2 \pi}{L^{2}} \sum_{\mathbf{k}} \Omega_{k_{x} k_{y}}^{\sigma},
$$

where, $L$ is the length of the sample. The phonon Hall conductivity formula, Eq. (5), is recasted into

$$
\kappa_{x y}=\frac{\hbar}{8 V T} \sum_{\mathbf{k}, \sigma \neq \sigma^{\prime}} f\left(\omega_{\sigma}\right)\left(\omega_{\sigma}+\omega_{\sigma^{\prime}}\right)^{2} \Omega_{k_{x} k_{y}}^{\sigma \sigma^{\prime}} .
$$

Here $V=L^{2} a$. The term $\left(\omega_{\sigma}+\omega_{\sigma^{\prime}}\right)^{2}$ relating to the phonon energy is an analog of the electrical charge term $e^{2}$ in the electron Hall effect, thus the phonon Hall conductivity Eq. (9) is similar to but different from the electron case because the phonon energy term can not be moved out from the summation. Although the formula is derived from the phonon transport in the crystal-lattice system, we note that the thermal Hall conductivity for the magnon Hall effect [19] can also be cast into the form of Eq. (9) with a different expression for the Berry curvature. Therefore, the Hall conductivity formula can be universally applicable to the thermal Hall effect in phonon and magnon systems without restriction for special lattice structures.

Without the Raman spin-phonon interaction, namely, $h=0, \Omega_{k_{x} k_{y}}^{\sigma \sigma^{\prime}}$ is zero everywhere and the phonon Hall conductivity vanishes. When a magnetic field is applied, the Berry curvature is nonzero, and consequently, the PHE appears. It is found that if the system exhibits symmetry satisfying $S D S^{-1}=D, S A S^{-1}=-A$ (e.g., mirror reflection symmetry), the phonon Hall conductivity is zero [4, 16]. This symmetry principle can also be
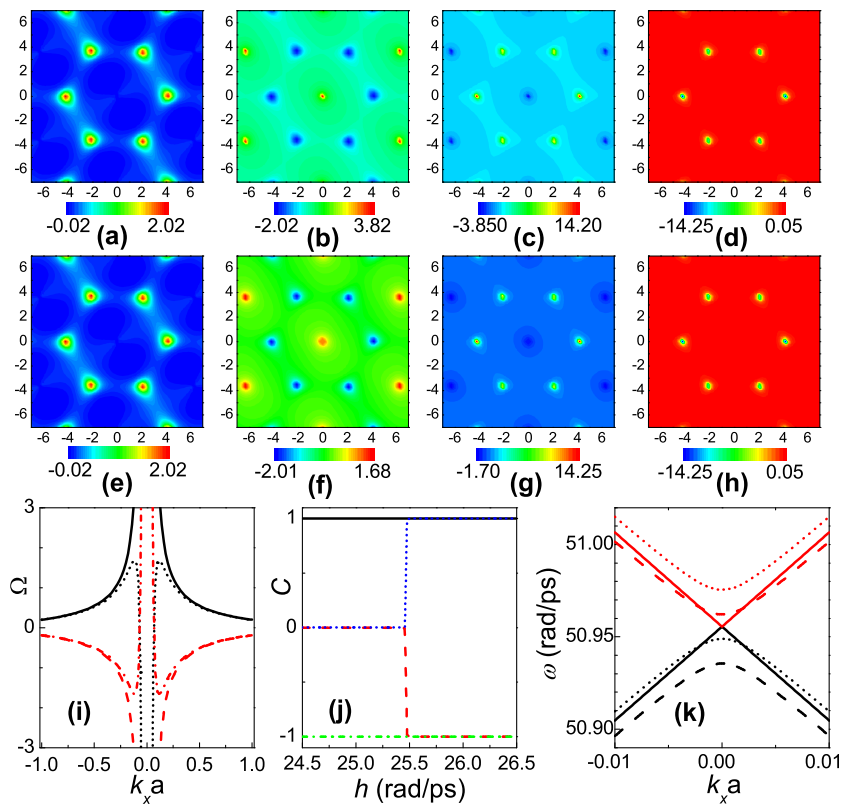

FIG. 2: (color online) (a)-(d) The contour map of Berry curvatures for bands $1-4$ at $h_{c-}=h_{c}-10^{-2} \mathrm{rad} / \mathrm{ps}$; (e)(h) The contour map of Berry curvatures for bands $1-4$ at $h_{c+}=h_{c}+10^{-2} \mathrm{rad} / \mathrm{ps}$. For (a)-(h), the horizontal and vertical axes correspond to wave vector $k_{x}$ and $k_{y}$, respectively. (i) $\Omega$ at different magnetic fields. The solid and dashed lines correspond to $\Omega^{2}$ and $\Omega^{3}$ at $h_{c-}$ respectively, while dotted and dash-dotted lines correspond to those at $h_{c+}$. (j) Chern numbers of four bands: $C^{1}$ (solid line), $C^{2}$ (dashed line), $C^{3}$ (dotted line), and $C^{4}$ (dash-dotted line). (k) The dispersion relation of band 2 and 3 at different magnetic fields in the vicinity of $h_{c}$. The dashed, solid and dotted lines correspond to the bands at $h_{c-}, h_{c}$, and $h_{c+}$, respectively. The lower three and upper three correspond to bands 2 and 3, respectively. $k_{y}=0$ in (i) and (k).

applied to the topological property of the phonon bands: we find that $\Omega_{k_{x} k_{y}}^{\sigma \sigma^{\prime}}=0$ provided that such symmetry exists, such as in the square lattice system. Whereas if such symmetry is broken for the dynamic matrix, the system can possess nontrivial Berry curvatures. In the system with the PHE, if the magnetic field changes, the Berry curvatures are quite different. However, we find that the associated topological Chern numbers remain constant integers with occasional jumps when $h$ is varied. Therefore, the Chern numbers given by Eq. (8) are topological invariant, which indeed illustrates the nontrivial topology of the phonon band structures. Although the Chern numbers are quantized to integers, the phonon Hall conductivity is not, due to the extra term $f\left(\omega_{\sigma}\right)\left(\omega_{\sigma}+\omega_{\sigma^{\prime}}\right)^{2}$. Thus, the analogy to the quantum Hall effect is incomplete.

In the vicinity of the critical magnetic field $h_{c}$, we find that the phase transition is indeed related to the abrupt change of the topology of band structures. The Berry curvatures for different bands near the critical magnetic 
field are illustrated in Fig. 2(a-h). We find that with an infinitesimal change of magnetic field around $h_{c}$, the Berry curvatures around the $\Gamma(\mathbf{k}=0)$ point of bands 2 and 3 are quite different, whereas those of band 1 and 4 remain unchanged. To illustrate the change of the Berry curvatures clearly, we plot the cross section of the Berry curvatures along the $k_{x}$ direction for bands 2 and 3 in Fig. 2(i), which shows explicitly that the Berry curvatures change dramatically above and below the critical magnetic field $h_{c}$. Below the critical point, the Berry curvature for band 2 in the vicinity of $\Gamma$ point contributes Berry phase $2 \pi$ ( $-2 \pi$ for band 3 ), which cancels that from $K$,and $K^{\prime}$ points, so that the Chern number is zero for bands 2 and 3, as indicated in Fig. 2(j). However, above the critical point, the sum of Berry curvature at $\Gamma$ point is zero, and only the monopole at $K$,and $K^{\prime}$ points contributes to Berry phase $(-2 \pi$ for band 2 and $2 \pi$ for band 3 ). Therefore, the Chern numbers jump from 0 to \pm 1 , as shown in Fig. 2(j). This jump indicates that the topology of the two bands suddenly changes at the critical magnetic field, which is responsible for the phase transition. From a calculation on the kagome lattice, which has been used to model many real materials [20], we also find qualitatively similar phase transitions due to the sudden change of topology, where the phonon Hall conductivity has three singularities of divergent first derivatives corresponding to three jumps of the Chern numbers.

To further investigate the mechanism of the abrupt change of the phonon band topology, we study the dispersion relation near the critical magnetic field. From Fig. 2(k), we can see that band 2 and 3 are going to touch with each other at the $\Gamma$ point if the magnetic field increases to $h_{c}$; at the critical magnetic field, the degeneracy occurs and the two bands possess the cone shape; above the critical point $h_{c}$, the two bands split up. Therefore, the difference between the two bands decreases below and increases above the critical point $h_{c}$. The property of the dispersion relation in the vicinity of the critical magnetic field directly affects the Berry curvature of the corresponding bands.

In summary, we have studied the PHE from a topological point of view. By looking at the phases of the polarization vectors of both the displacements and conjugate momenta as a function of the wave vector, a Berry curvature can be defined uniquely for each band. This Berry curvature can be used to calculate the phonon Hall conductivity. Because of the nature of phonons, the phonon Hall conductivity, which is not directly proportional to the Chern number, is not quantized. However, the quantization effect, in the sense of discontinuous jumps in Chern numbers, manifests itself in the phonon Hall conductivity as a singularity of the first derivative with respect to the magnetic field.

The topological approach for phonon Hall conductivity proposed here is general and can be applied to the real materials in low temperatures where the thermal trans- port is ballistic. It can also be applied to the magnon Hall effect discovered recently [19]. Phase transition in the PHE, explained from topological nature and dispersion relations, can also be generalized to study the phase transition in other Hall effects and/or nonequilibrium transport. In line with recently reported Berry-phaseinduced heat pumping [21] and the Berry-phase contribution of molecular vibrational instability [22], we hope our present results do invigorate the studies aimed at uncovering intriguing Berry phase effects and topological properties in phonon transport, which will enrich further the discipline of phononics.

L.Z. thanks Bijay Kumar Agarwalla and Jie Chen for fruitful discussions. This project is supported in part by Grants No. R-144-000-257-112 and No. R-144-000-222646 of NUS.

* Electronic address: zhanglifa@nus.edu.sg

† Electronic address: renjie@nus.edu.sg

¥ Electronic address: phywjs@nus.edu.sg

$\S$ Electronic address: phylibw@nus.edu.sg

[1] L. Wang and B. Li, Physics World 21, No.3, 27 (2008).

[2] B. Li, L. Wang and G Casati, Phys. Rev. Lett 93184301 (2004); C. W Chang, D. Okawa, A. Majumdar, and A. Zettl, Science 314, 1121 (2006).

[3] B. Li, L. Wang and G. Casati, Appl. Phys. Lett. 88, 143501 (2006).

[4] L. Wang and B. Li, Phys. Rev. Lett 99, 177208 (2007).

[5] L. Wang and B. Li, Phys. Rev. Lett. 101, 267203 (2008).

[6] C. Strohm, G. L. J. A. Rikken, and P. Wyder, Phys. Rev. Lett. 95, 155901 (2005).

[7] A. V. Inyushkin and A. N. Taldenkov, JETP Lett. 86, 379 (2007).

[8] L. Sheng, D. N. Sheng, and C. S. Ting, Phys. Rev. Lett. 96, 155901 (2006).

[9] Y. Kagan and L. A. Maksimov, Phys. Rev. Lett. 100, 145902 (2008).

[10] J.-S. Wang and L. Zhang, Phys. Rev. B. 80, 012301 (2009); L. Zhang, J.-S. Wang, and B. Li, New J. Phys. 11, 113038 (2009).

[11] D. J. Thouless, M. Kohmoto, M. P. Nightingale, and M. den Nijs, Phys. Rev. Lett. 49, 405 (1982).

[12] N. Nagaosa, J. Sinova, S. Onoda, A. H. MacDonald, and N. P. Ong, Rev. Mod. Phys. 82, 1539 (2010).

[13] M. Koenig, et al. J. Phys. Soc. Jpn. 77, 031007 (2008).

[14] D. Xiao, M.-C. Chang, Q. Niu, Rev. Mod. Phys. 82, 1959 (2010).

[15] A. Holz, Il Nuovo Cimento B 9, 83 (1972).

[16] See the detailed discussion in supplementary information.

[17] R. J. Hardy, Phys. Rev. 132, 168 (1963).

[18] G. D. Mahan, Many-Particle Physics 3rd ed. (Kluwer Academic, New York, 2000).

[19] H. Katsura, N. Nagaosa, and P. A. Lee, Phys. Rev. Lett. 104, 066403 (2010); Y. Onose, T. Ideue, H. Katsura, Y. Shiomi, N. Nagaosa, Y. Tokura, Science 329, 297 (2010).

[20] I. Syozi, Prog. Theor. Phys. 6, 306 (1951); M. Takano, T. Shinjo, M. Kiyama, T. Takada, J. Phys. Soc. Jpn. 25, 902 (1968); M. Wolf, K. D. Schotte, J. Phys. A 21, 2195 
(1988); V. Elser, Phys. Rev. Lett. 62, 2405 (1989); C. Broholm, G. Appli, G. P. Espinosa, A. S. Cooper, Phys. Rev. Lett. 65, 3173 (1990).

[21] J. Ren, P. Hänggi, and B. Li, Phys. Rev. Lett. 104, 170601 (2010).

[22] J.-T. Lü, M. Brandbyge, and P. Hedegård, Nano Lett. 10, 1657 (2010).

SUPPLEMENTARY INFORMATION FOR “TOPOLOGICAL NATURE OF PHONON HALL EFFECT"

ABSTRACT

In this supplementary material, we discuss the origination of the Hamiltonian [Eq. (1) in the main text] in the first section; then we present the detailed derivation of the general formula of phonon Hall conductivity in terms of Berry curvature, in which we also give the explicit expression for the dynamic matrix and give the proof for the symmetry principle. Finally, we discuss the numerical calculation for Chern numbers.

\section{DISCUSSION ON THE HAMILTONIAN}

In the presence of a magnetic field, according to Ref. [1], the kinetic energy of each site in ionic crystal lattices without free charges is expressed as:

$$
T_{\alpha}=\frac{1}{2} m_{\alpha}\left|\dot{\mathbf{r}}_{\alpha}\right|^{2}=\frac{1}{2 m_{\alpha}}\left|\mathbf{p}_{\alpha} \sqrt{m_{\alpha}}-q_{\alpha} \mathbf{A}_{\alpha}\right|^{2},
$$

where, $\mathbf{r}_{\alpha}=\mathbf{R}_{\alpha}+\mathbf{u}_{\alpha} / \sqrt{m_{\alpha}}, \mathbf{R}_{\alpha}$ is the equilibrium coordinate of the ion at site $\alpha$, and $\mathbf{u}_{\alpha}$ denotes the displacement multiplied by the square root of the ion mass $m_{\alpha} \cdot \mathbf{p}_{\alpha}$ is the corresponding momentum divided by the square root of mass $m_{\alpha} . q_{\alpha}$ is the ionic charge at site $\alpha . \mathbf{A}_{\alpha}$ denotes the electromagnetic vector potential, which, using the Lorenz gauge condition, can be related to the ionic displacement as [1]

$$
\mathbf{A}_{\alpha}=\frac{1}{2} \mathbf{B} \times \mathbf{u}_{\alpha} / \sqrt{m_{\alpha}}
$$

Thus, Eq. (S1) is recasted as:

$$
T_{\alpha}=\frac{1}{2}\left|\mathbf{p}_{\alpha}-\frac{q_{\alpha}}{2 m_{\alpha}} \mathbf{B} \times \mathbf{u}_{\alpha}\right|^{2} .
$$

If the magnetic field with magnitude $B$ is applied along $z$ direction and we only consider the two-dimensional ( $x$ and $y$ direction) motion of the system, then the kinetic energy of ion $\alpha$ can be expressed (it is straightforward to generalize 
to high dimentions) as:

$$
T_{\alpha}=\frac{1}{2}\left(p_{\alpha}-\Lambda_{\alpha} u_{\alpha}\right)^{T}\left(p_{\alpha}-\Lambda_{\alpha} u_{\alpha}\right)
$$

where $p_{\alpha}=\left(p_{\alpha x}, p_{\alpha y}\right)^{T}, u_{\alpha}=\left(u_{\alpha x}, u_{\alpha y}\right)^{T}$, and $\Lambda_{\alpha}=\left(\begin{array}{cc}0 & h_{\alpha} \\ -h_{\alpha} & 0\end{array}\right)$, where $h_{\alpha}=-q_{\alpha} B /\left(2 m_{\alpha}\right)$. Note that there are both positive and negative ions in one unit cell. For a general ionic paramagnetic dielectric, mostly, the mass of the positive ion is larger than that of the negative one. For instance, in the experimental sample $\mathrm{Tb}_{3} \mathrm{Ga}_{5} \mathrm{O}_{12}$, the ratio $m(+q) / m(-q)$ is about 4.3 in one unit cell. Therefore the negative ions will dominate in the contribution to $h_{\alpha}$, which makes $h_{\alpha}$ have the same sign as that of the applied magnetic field $B$. Under the mean-field approximation, we can set $h_{\alpha}=h$, which is site-independent and is proportional to the magnitude of the applied magnetic field.

Combining the kinetic energy with the harmonic inter-potential energy, we can write the whole Hamiltonian as

$$
H=\frac{1}{2}(p-\tilde{A} u)^{T}(p-\tilde{A} u)+\frac{1}{2} u^{T} K u,
$$

where $\tilde{A}$ is an antisymmetric real matrix with block-diagonal elements $\Lambda_{\alpha}$. u and $p$ are column vectors denoting displacements and momenta respectively, for all the degrees of freedom. $K$ indicates the force constant matrix. Finally, after the rearrangement, we have

$$
H=\frac{1}{2} p^{T} p+\frac{1}{2} u^{T}\left(K-\tilde{A}^{2}\right) u+u^{T} \tilde{A} p
$$

which is exactly the second row of Eq. (1) in the text.

The Hamiltonian Eq. (S6) [Eq.(1) in the main text] is essentially the same as that used in Ref. [2 [5] resulting from the phenomenological Raman interaction. The only difference is the term proportional to $\tilde{A}^{2}$ which makes the above Hamiltonian positive definite. The Raman interaction, proposed to study spin-phonon interactions (SPI) based on quantum theory and fundamental symmetries [6 [8], can be expressed as

$$
H_{I}=g \mathbf{s} \cdot(\mathbf{u} \times \mathbf{p}) .
$$

Here, $g$ denotes a positive coupling constant, and $\mathbf{s}$ is the isospin for the lowest quasidoublet. In the presence of a magnetic field $\mathbf{B}$, each site has a magnetization $\mathbf{M}$. For isotropic SPI, the isospin $\mathbf{s}$ is parallel to $\mathbf{M}$, and the ensemble average of the isospin is proportional to the magnetization, which can be expressed as $\langle\mathbf{s}\rangle=c \mathbf{M}$ with $c$ the proportionality coefficient (Ref. [2 [5]). In the mean-field approximation, the Raman type SPI reduces to

$$
H_{I}=\mathbf{h} \cdot(\mathbf{u} \times \mathbf{p}),
$$

where $\mathbf{h}=g c \mathbf{M}$, and $\mathbf{M}$ is proportional to the magnetic field $\mathbf{B}$. If the magnetic field is applied along the $z$ direction, then the SPI can be written as

$$
H_{I}=u^{T} \tilde{A} p
$$

By treating the phonon system under harmonic approximation, the total Hamiltonian for the whole lattice can be written as (Ref. 2[4])

$$
H=\frac{1}{2} p^{T} p+\frac{1}{2} u^{T} K u+u^{T} \tilde{A} p
$$

Note that this Hamiltonian Eq. (S10) is not positive definite. In Ref.[4], the authors added an arbitrary onsite potential in order to make the Hamiltonian positive definite. However, in the calculation of phonon Hall effect for the four-terminal junctions, such non-positive-definite Hamiltonian does not cause any problem because the thermal junctions will stabilize the system [5].

From the first physical picture of spin-phonon interaction in ionic crystal lattice with an applied magnetic field (Eq. ?? $(\mathbf{S 6})$, the additional term proportional to $\tilde{A}^{2}$ emerges naturally to make the Hamiltonian positive definite. Therefore, in this work we choose the positive definite Hamiltonian Eq. (S6) [Eq. (1) in the main text]. 


\section{PHONON HALL CONDUCTIVITY FROM GREEN-KUBO FORMULA}

The Hamiltonian Eq. (S6) is quadratic in $u$ and $p$, and we can write the equation of motion as

$$
\begin{aligned}
& \dot{p}=-\left(K-\tilde{A}^{2}\right) u-\tilde{A} p \\
& \dot{u}=p-\tilde{A} u .
\end{aligned}
$$

The equation of motion for the coordinate is,

$$
\ddot{u}+2 \tilde{A} \dot{u}+\tilde{A}^{2} u+\left(K-\tilde{A}^{2}\right) u=0 .
$$

Since the lattice is periodic, we can apply the Bloch's theorem $u_{l}=\epsilon e^{i\left(\mathbf{R}_{l} \cdot \mathbf{k}-\omega t\right)}$. The polarization vector $\epsilon$ satisfies

$$
\left[(-i \omega+A)^{2}+D\right] \epsilon=0
$$

where $D(\mathbf{k})=-A^{2}+\sum_{l^{\prime}} K_{l, l^{\prime}} e^{i\left(\mathbf{R}_{l^{\prime}}-\mathbf{R}_{l}\right) \cdot \mathbf{k}}$ denotes the dynamic matrix and $A$ is block diagonal with elements $\Lambda$. $D, K_{l, l^{\prime}}$, and $A$ are all $n d \times n d$ matrices, where $n$ is the number of particles in one unit cell and $d$ is the dimension of the motion.

To calculate the dynamic matrix $D(\mathbf{k})$, we give an example for the two-dimensional honeycomb lattice, where $n=2, d=2$. We only consider the nearest neighbor interaction. The spring constant matrix along $x$ direction is

$$
K_{x}=\left(\begin{array}{cc}
K_{L} & 0 \\
0 & K_{T}
\end{array}\right) .
$$

$K_{L}=0.144 \mathrm{eV} /\left(\mathrm{u} \AA^{2}\right)$ is the longitudinal spring constant and the transverse one $K_{T}$ is 4 times smaller. The unit cell lattice vectors are $(a, 0)$ and $(a / 2, a \sqrt{3} / 2)$ with $a=1 \AA$.

To obtain the explicit formula for the dynamic matrix, we first define a rotation operator in two dimensions as:

$$
U(\theta)=\left(\begin{array}{cc}
\cos \theta & -\sin \theta \\
\sin \theta & \cos \theta
\end{array}\right)
$$

The three kinds of spring-constant matrices between two atoms are $K_{01}=U(\pi / 2) K_{x} U(-\pi / 2), \quad K_{02}=$ $U(\pi / 6) K_{x} U(-\pi / 6), K_{03}=U(-\pi / 6) K_{x} U(\pi / 6)$, which are $2 \times 2$ matrices. Then we can obtain the on-site springconstant matrix and the four spring-constant matrices between the unit cell and its four nearest neighbors as:

$$
\begin{gathered}
K_{0}=\left(\begin{array}{cc}
K_{01}+K_{02}+K_{03} & -K_{02} \\
-K_{02} & K_{01}+K_{02}+K_{03}
\end{array}\right), \\
K_{1}=\left(\begin{array}{cc}
0 & 0 \\
-K_{03} & 0
\end{array}\right), \quad K_{2}=\left(\begin{array}{cc}
0 & 0 \\
-K_{01} & 0
\end{array}\right), \quad K_{3}=\left(\begin{array}{cc}
0 & -K_{03} \\
0 & 0
\end{array}\right), \quad K_{4}=\left(\begin{array}{cc}
0 & -K_{01} \\
0 & 0
\end{array}\right),
\end{gathered}
$$

which are $4 \times 4$ matrices. Finally we can obtain the $4 \times 4$ dynamic matrix $D(\mathbf{k})$ as

$$
D(\mathbf{k})=-A^{2}+K_{0}+K_{1} e^{i k_{x}}+K_{2} e^{i\left(k_{x} / 2+\sqrt{3} k_{y} / 2\right)}+K_{3} e^{-i k_{x}}+K_{4} e^{-i\left(k_{x} / 2+\sqrt{3} k_{y} / 2\right)},
$$

where, $A^{2}=-h^{2} \cdot I$, and $I$ denotes the $4 \times 4$ identity matrix.

Equation (S14) can be written in a form as a standard eigen-problem given in Eq. (3) in the main text, if we rewrite the equations of motion. Using Bloch theorem, Eqs. (S11) and (S12) can be recasted as:

$$
i \frac{\partial}{\partial t} x=H_{\mathrm{eff}} x, \quad H_{\mathrm{eff}}=i\left(\begin{array}{cc}
-A & -D \\
I & -A
\end{array}\right) .
$$

$x=(\mu, \epsilon)^{T}$ is the polarization vector, where column vectors $\mu$ and $\epsilon$ are associated with the momenta and coordinates respectively. Therefore, the right eigenvector and left eigenvector satisfy:

$$
H_{\mathrm{eff}} x_{\sigma}=\omega_{\sigma} x_{\sigma}, \quad \tilde{x}_{\sigma}^{T} H_{\mathrm{eff}}=\omega_{\sigma} \tilde{x}_{\sigma}^{T} .
$$


where the right eigenvector $x_{\sigma}=\left(\mu_{\sigma}, \epsilon_{\sigma}\right)^{T}$, the left eigenvector $\tilde{x}_{\sigma}^{T}=\left(\epsilon_{\sigma}^{\dagger},-\mu_{\sigma}^{\dagger}\right) /\left(-2 i \omega_{\sigma}\right)$, and $\sigma$ indicates the branch index. Because the effective Hamiltonian $H_{\text {eff }}$ is not hermitian, the orthonormal condition then holds between the left and right eigenvectors. The eigenmodes can be normalized as $\tilde{x}_{\sigma}^{T} x_{\sigma}=1$, which is equivalent to [4]

$$
\epsilon_{\sigma}^{\dagger} \epsilon_{\sigma}+\frac{i}{\omega_{\sigma}} \epsilon_{\sigma}^{\dagger} A \epsilon_{\sigma}=1
$$

To solve the eigensystem, we require the following relations:

$$
\epsilon_{-\mathbf{k},-\sigma}^{*}=\epsilon_{\mathbf{k}, \sigma} ; \omega_{-\mathbf{k},-\sigma}=-\omega_{\mathbf{k}, \sigma} .
$$

In the following, we use $k=(\mathbf{k}, \sigma)$ to specify both the wavevector and the phonon branch. By taking into account only positive eigen-modes $(\omega>0)$, displacement and momentum operators are taken in the second quantization form:

$$
\begin{aligned}
& u_{l}=\sum_{k} \epsilon_{k} e^{i\left(\mathbf{R}_{l} \cdot \mathbf{k}-\omega_{k} t\right)} \sqrt{\frac{\hbar}{2 \omega_{k} N}} a_{k}+\text { h.c. }, \\
& p_{l}=\sum_{k} \mu_{k} e^{i\left(\mathbf{R}_{l} \cdot \mathbf{k}-\omega_{k} t\right)} \sqrt{\frac{\hbar}{2 \omega_{k} N}} a_{k}+\text { h.c. },
\end{aligned}
$$

where $\sigma>0, a_{k}$ is the annihilation operator, and h.c. stands for hermitian conjugate. The momentum and displacement polarization vectors are related through $\mu_{k}=-i \omega_{k} \epsilon_{k}+A \epsilon_{k}$. We can verify that the canonical commutation relations are satisfied: $\left[u_{l}, p_{l^{\prime}}^{T}\right]=i \hbar \delta_{l, l^{\prime}} I$, and $H=\sum_{k} \hbar \omega_{k}\left(a_{k}^{\dagger} a_{k}+1 / 2\right)$.

The energy current density is defined as [9]:

$$
\mathbf{J}=\frac{1}{2 V} \sum_{l, l^{\prime}}\left(\mathbf{R}_{l}-\mathbf{R}_{l^{\prime}}\right) u_{l}^{T} K_{l, l^{\prime}} \dot{u}_{l^{\prime}},
$$

where $V$ is the total volume of $N$ unit cells. The current density vector can be expressed in terms of the creation/annihilation operators as

$$
\begin{aligned}
& \mathbf{J}=\mathbf{J}_{1}\left(a^{\dagger} a\right)+\mathbf{J}_{2}\left(a^{\dagger} a^{\dagger}, a a\right) ; \\
& \mathbf{J}_{1}=\frac{\hbar}{4 V} \sum_{k, k^{\prime}}\left(\sqrt{\frac{\omega_{k}}{\omega_{k^{\prime}}}}+\sqrt{\frac{\omega_{k^{\prime}}}{\omega_{k}}}\right) \epsilon_{k}^{\dagger} \frac{\partial D(\mathbf{k})}{\partial \mathbf{k}} \epsilon_{k^{\prime}} a_{k}^{\dagger} a_{k^{\prime}} \delta_{\mathbf{k}, \mathbf{k}^{\prime}} e^{i\left(\omega_{k}-\omega_{k^{\prime}}\right) t} \\
& \mathbf{J}_{2}=\frac{\hbar}{4 V} \sum_{k, k^{\prime}} \sqrt{\frac{\omega_{k^{\prime}}}{\omega_{k}}}\left(\epsilon_{k}^{\dagger} \frac{\partial D(\mathbf{k})}{\partial \mathbf{k}} \epsilon_{k^{\prime}}^{*} a_{k}^{\dagger} a_{k^{\prime}}^{\dagger} e^{i\left(\omega_{k}+\omega_{k^{\prime}}\right) t}+\epsilon_{k}^{T} \frac{\partial D^{*}(\mathbf{k})}{\partial \mathbf{k}} \epsilon_{k^{\prime}} a_{k} a_{k^{\prime}} e^{-i\left(\omega_{k}+\omega_{k^{\prime}}\right) t}\right) \delta_{\mathbf{k},-\mathbf{k}^{\prime}} .
\end{aligned}
$$

We note that the $a^{\dagger} a^{\dagger}$ and $a a$ terms also contribute to the off-diagonal elements of the thermal conductivity tensor, although they have no contribution to the average energy current. Based on the expression of heat current, the phonon Hall conductivity can be obtained through the Green-Kubo formula [10]:

$$
\kappa_{x y}=\frac{V}{\hbar T} \int_{0}^{\hbar /\left(k_{B} T\right)} d \lambda \int_{0}^{\infty} d t\left\langle J^{x}(-i \lambda) J^{y}(t)\right\rangle_{\mathrm{eq}},
$$

where the average is taken over the equilibrium ensemble with Hamiltonian $H$. Substituting the expression $\mathbf{J}$ into Eq. (S27), the phonon Hall conductivity is obtained as

$$
\begin{aligned}
\kappa_{x y} & =\kappa_{x y}^{(1)}+\kappa_{x y}^{(2)} ; \\
\kappa_{x y}^{(1)} & =\frac{V}{\hbar T} \int_{0}^{\hbar /\left(k_{B} T\right)} d \lambda \int_{0}^{\infty} d t\left\langle J_{1}^{x}(-i \lambda) J_{1}^{y}(t)\right\rangle_{\mathrm{eq}} ; \\
\kappa_{x y}^{(2)} & =\frac{V}{\hbar T} \int_{0}^{\hbar /\left(k_{B} T\right)} d \lambda \int_{0}^{\infty} d t\left\langle J_{2}^{x}(-i \lambda) J_{2}^{y}(t)\right\rangle_{\mathrm{eq}} .
\end{aligned}
$$

Note that the averages of the cross terms $\left\langle J_{1}^{x}(-i \lambda) J_{2}^{y}(t)\right\rangle_{\text {eq }}$ and $\left\langle J_{2}^{x}(-i \lambda) J_{1}^{y}(t)\right\rangle_{\text {eq }}$ are zero.

First we calculate the term $\kappa_{a b}^{(1)}$. Combining the result

$$
\left\langle a_{i}^{\dagger} a_{j} a_{k}^{\dagger} a_{l}\right\rangle_{\mathrm{eq}}=f_{i} f_{k} \delta_{i j} \delta_{k l}+f_{i}\left(f_{j}+1\right) \delta_{i l} \delta_{j k},
$$

where $f_{i}=\left(e^{\beta \hbar \omega_{i}}-1\right)^{-1}$ is the Bose distribution function, with the result

$$
\sum_{\mathbf{k}} \epsilon_{\sigma}^{\dagger} \frac{\partial D}{\partial k_{\alpha}} \epsilon_{\sigma}=-2 i \sum_{\mathbf{k}} \omega_{\sigma} \tilde{x}_{\sigma}^{T} \frac{\partial H_{\mathrm{eff}}}{\partial k_{\alpha}} x_{\sigma}=-2 i \sum_{\mathbf{k}} \omega_{\sigma} \frac{\partial \omega_{\sigma}}{\partial k_{\alpha}}=0, \quad(\alpha=x, y)
$$


which is obtained by differentiating the Eq. (S20) (Eq. (3) in the main text) and $\tilde{x}_{\sigma}^{T} x_{\sigma}=1$, we obtain

$$
\kappa_{x y}^{(1)}=\frac{\hbar}{16 V T} \sum_{\mathbf{k}, \sigma>0, \sigma^{\prime}>0}\left[f\left(\omega_{\sigma}\right)-f\left(\omega_{\sigma}^{\prime}\right)\right]\left(\omega_{\sigma}+\omega_{\sigma}^{\prime}\right)^{2} \frac{i}{\omega_{\sigma} \omega_{\sigma^{\prime}}} \frac{\epsilon_{\sigma}^{\dagger} \frac{\partial D}{\partial k_{x}} \epsilon_{\sigma^{\prime}} \epsilon_{\sigma^{\prime}}^{\dagger} \frac{\partial D}{\partial k_{y}} \epsilon_{\sigma}}{\left(\omega_{\sigma}-\omega_{\sigma^{\prime}}\right)^{2}} .
$$

Because of Eq. (S22) and the following property:

$$
D_{a b}(-\mathbf{k})=D_{a b}^{*}(\mathbf{k})=D_{b a}(\mathbf{k}),
$$

we can transform from the positive-frequency bands to the negative-frequency band, and obtain

$$
\kappa_{x y}^{(1)}=\frac{\hbar}{8 V T} \sum_{\mathbf{k}, \sigma \sigma^{\prime}>0}\left[f\left(\omega_{\sigma}\right)-f\left(\omega_{\sigma^{\prime}}\right)\right]\left(\omega_{\sigma}+\omega_{\sigma^{\prime}}\right)^{2} \frac{i}{4 \omega_{\sigma} \omega_{\sigma^{\prime}}} \frac{\epsilon_{\sigma}^{\dagger} \frac{\partial D}{\partial k_{x}} \epsilon_{\sigma^{\prime}} \epsilon_{\sigma^{\prime}}^{\dagger} \frac{\partial D}{\partial k_{y}} \epsilon_{\sigma}}{\left(\omega_{\sigma}-\omega_{\sigma^{\prime}}\right)^{2}} .
$$

Here, $\sigma, \sigma^{\prime}$ can be both positive or negative.

Second, we calculate $\kappa_{a b}^{(2)}$. Utilizing the results

$$
\begin{aligned}
& \left\langle a_{i}^{\dagger} a_{j}^{\dagger} a_{k} a_{l}\right\rangle_{\mathrm{eq}}=f_{i} f_{j}\left(\delta_{i k} \delta_{j l}+\delta_{i l} \delta_{j k}\right) ; \\
& \left\langle a_{i} a_{j} a_{k}^{\dagger} a_{l}^{\dagger}\right\rangle_{\mathrm{eq}}=\left(1+f_{i}\right)\left(1+f_{j}\right)\left(\delta_{i k} \delta_{j l}+\delta_{i l} \delta_{j k}\right),
\end{aligned}
$$

and the relation $f(-\omega)=-1-f(\omega)$, after some algebraic derivation similar to the above, we obtain

$$
\kappa_{x y}^{(2)}=\frac{\hbar}{8 V T} \sum_{\mathbf{k}, \sigma \sigma^{\prime}<0}\left[f\left(\omega_{\sigma}\right)-f\left(\omega_{\sigma^{\prime}}\right)\right]\left(\omega_{\sigma}+\omega_{\sigma^{\prime}}\right)^{2} \frac{i}{4 \omega_{\sigma} \omega_{\sigma^{\prime}}} \frac{\epsilon_{\sigma}^{\dagger} \frac{\partial D}{\partial k_{x}} \epsilon_{\sigma^{\prime}} \epsilon_{\sigma^{\prime}}^{\dagger} \frac{\partial D}{\partial k_{y}} \epsilon_{\sigma}}{\left(\omega_{\sigma}-\omega_{\sigma^{\prime}}\right)^{2}} .
$$

Therefore, the total phonon Hall conductivity can be written as

$$
\kappa_{x y}=\frac{\hbar}{8 V T} \sum_{\mathbf{k}, \sigma \neq \sigma^{\prime}}\left[f\left(\omega_{\sigma}\right)-f\left(\omega_{\sigma^{\prime}}\right)\right]\left(\omega_{\sigma}+\omega_{\sigma^{\prime}}\right)^{2} \frac{i}{4 \omega_{\sigma} \omega_{\sigma^{\prime}}} \frac{\epsilon_{\sigma}^{\dagger} \frac{\partial D}{\partial k_{x}} \epsilon_{\sigma^{\prime}} \epsilon_{\sigma^{\prime}}^{\dagger} \frac{\partial D}{\partial k_{y}} \epsilon_{\sigma}}{\left(\omega_{\sigma}-\omega_{\sigma^{\prime}}\right)^{2}} .
$$

We can prove $\kappa_{x y}=-\kappa_{y x}$, such that

$$
\begin{gathered}
\kappa_{x y}=\frac{\hbar}{16 V T} \sum_{\mathbf{k}, \sigma \neq \sigma^{\prime}}\left[f\left(\omega_{\sigma}\right)-f\left(\omega_{\sigma^{\prime}}\right)\right]\left(\omega_{\sigma}+\omega_{\sigma^{\prime}}\right)^{2} \Omega_{k_{x} k_{y}}^{\sigma \sigma^{\prime}}, \\
\Omega_{k_{x} k_{y}}^{\sigma \sigma^{\prime}}=\frac{i}{4 \omega_{\sigma} \omega_{\sigma^{\prime}}} \frac{\epsilon_{\sigma}^{\dagger} \frac{\partial D}{\partial k_{x}} \epsilon_{\sigma^{\prime}} \epsilon_{\sigma^{\prime}}^{\dagger} \frac{\partial D}{\partial k_{y}} \epsilon_{\sigma}-\epsilon_{\sigma}^{\dagger} \frac{\partial D}{\partial k_{y}} \epsilon_{\sigma^{\prime}} \epsilon_{\sigma^{\prime}}^{\dagger} \frac{\partial D}{\partial k_{x}} \epsilon_{\sigma}}{\left(\omega_{\sigma}-\omega_{\sigma^{\prime}}\right)^{2}} .
\end{gathered}
$$

Because of $\Omega_{k_{x} k_{y}}^{\sigma \sigma^{\prime}}=-\Omega_{k_{x} k_{y}}^{\sigma^{\prime} \sigma}$, the phonon Hall conductivity can be written eventually as

$$
\kappa_{x y}=\frac{\hbar}{8 V T} \sum_{\mathbf{k}, \sigma \neq \sigma^{\prime}} f\left(\omega_{\sigma}\right)\left(\omega_{\sigma}+\omega_{\sigma^{\prime}}\right)^{2} \Omega_{k_{x} k_{y}}^{\sigma \sigma^{\prime}}
$$

where $V$ is the total volume of $N=N_{L}^{2}$ unit cells. In the above formula, the phonon branch $\sigma$ includes both positive and negative values without restriction. We start with the positive frequency bands to derive the conductivity formula. Through some transformations, we finally obtain the simplified formula for phonon Hall conductivity which combines the contribution from all the frequency bands. The formula Eq. (S38) is different from that given in Ref. [4]. In Ref. [4] the contribution for phonon Hall conductivity from $J_{2}$ was omitted, which is incorrect.

From the Eq. (S14), we obtain

$$
\epsilon_{-\mathbf{k}, \sigma}^{*}(-A)=\epsilon_{\mathbf{k}, \sigma}(A) ; \omega_{-\mathbf{k}, \sigma}=\omega_{\mathbf{k}, \sigma},
$$

and because of $D(\mathbf{k})=D^{*}(-\mathbf{k}), \epsilon_{\sigma}^{T} \frac{\partial D^{*}}{\partial k_{x}} \epsilon_{\sigma^{\prime}}^{*}=\epsilon_{\sigma^{\prime}}^{\dagger} \frac{\partial D}{\partial k_{x}} \epsilon_{\sigma}$, we have

$$
\Omega_{k_{x} k_{y}}^{\sigma \sigma^{\prime}}(\mathbf{k},-A)=\Omega_{k_{y} k_{x}}^{\sigma \sigma^{\prime}}(-\mathbf{k}, A)=-\Omega_{k_{x} k_{y}}^{\sigma \sigma^{\prime}}(-\mathbf{k}, A) .
$$



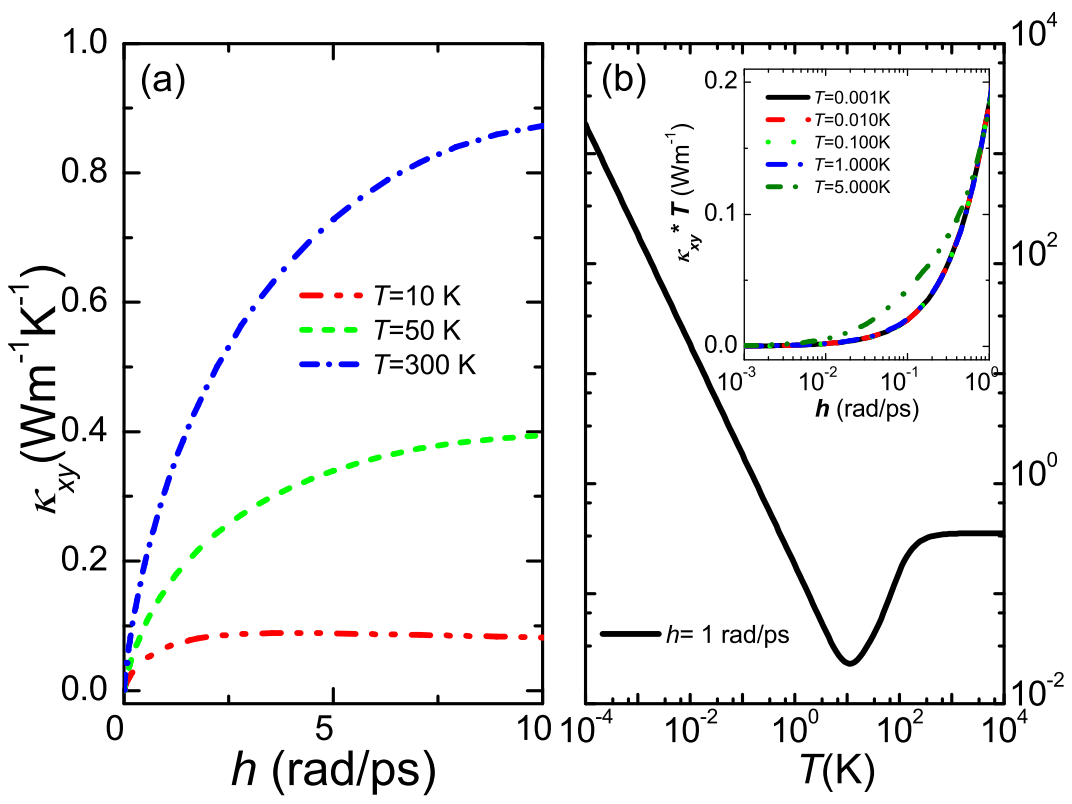

FIG. S1: (color online) (a) Phonon Hall conductivity vs applied magnetic field for two-dimensional honeycomb lattice. (b) Phonon Hall conductivity vs temperature at fixed magnetic field $h=1 \mathrm{rad} / \mathrm{ps}$. The inset of (b) shows the product of phonon Hall conductivity and temperature $\kappa_{x y} T$ vs magnetic field $h$ for different temperatures.

So we obtain

$$
\kappa_{x y}(-A)=\kappa_{y x}(A)=-\kappa_{x y}(A) .
$$

The Onsager reciprocal relations are satisfied.

If the system possesses the symmetry which satisfies

$$
S D S^{-1}=D, S A S^{-1}=-A,
$$

where $S$ represents any symmetric operation, and from Eq. (S14), we obtain

$$
S \epsilon(A)=\epsilon(-A) \text {. }
$$

Using the definition of the dynamic matrix $D=-A^{2}+\sum_{l^{\prime}} K_{l, l^{\prime}} e^{i\left(\mathbf{R}_{l^{\prime}}-\mathbf{R}_{l}\right) \cdot \mathbf{k}}$ and $S D S^{-1}=D$, we can obtain

$$
S \frac{\partial D}{\partial k_{\alpha}} S^{-1}=\frac{\partial D}{\partial k_{\alpha}}, \quad(\alpha=x, y)
$$

Inserting $S^{-1} S=I$ into Eq.(S37), we obtain

$$
\Omega_{k_{x} k_{y}}^{\sigma \sigma^{\prime}}(-A)=\Omega_{k_{x} k_{y}}^{\sigma \sigma^{\prime}}(A)
$$

Then it is easy to obtain $\kappa_{x y}(-A)=\kappa_{x y}(A)$, and because of the Onsager relation, one can easily obtain that

$$
\kappa_{x y}=0, \text { if } S D S^{-1}=D, S A S^{-1}=-A .
$$

Fig. S1 (a) shows the phonon Hall conductivity with magnetic field for different temperatures. In the weak magnetic field range, the phonon Hall conductivity $k_{x y}$ is proportional to the magnetic field, which is consistent with all the experimental and theoretical results. We plot phonon Hall conductivity with a large range of temperatures in Fig. [S1(b). At very low temperatures, the phonon Hall conductivity is proportional to $1 / T$. $k_{x y} T$ will be constant for different temperatures lower than $1 K$. This is due to the contribution from $\kappa_{x y}^{(2)}$ : if $T \rightarrow 0,1+f \rightarrow 1$, then the conductivity linear with $1 / T$ tends infinity. While the longitudinal thermal conductivity $\kappa_{x x}$ is infinite for any temperature [4], thus when $T \rightarrow 0$, the transverse Hall conductivity, $\kappa_{x y} \rightarrow \infty$, has the ballistic property similar to the longitudinal one. If temperature is very high, all the modes contribute to the thermal transport, and $f \simeq k_{B} T /(\hbar \omega)$, then the phonon Hall conductivity becomes a constant, which can be seen in Fig. S1(b). 


\section{THE BERRY PHASE AND BERRY CURVATURE}

Using the similar method proposed by Berry[11], we derive the Berry phase and Berry curvature in the following. Starting from

$$
i \frac{\partial}{\partial t} x(t)=H_{\mathrm{eff}} x(t)
$$

and substituting

$$
x(t)=e^{i \gamma_{\sigma}(t)-i \int_{0}^{t} d t^{\prime} \omega_{\sigma}\left(\mathbf{k}\left(t^{\prime}\right)\right)} x_{\sigma}(\mathbf{k}(t))
$$

we can obtain the Berry phase across the Brillouin zone as

$$
\gamma_{\sigma}=\oint \mathbf{A}^{\sigma}(\mathbf{k}) d \mathbf{k}, \quad \mathbf{A}^{\sigma}(\mathbf{k})=i \tilde{x}_{\sigma}^{T} \frac{\partial}{\partial \mathbf{k}} x_{\sigma} .
$$

Here $x_{\sigma}, \tilde{x}_{\sigma}^{T}$ correspond to the right and left eigenvectors, and $\tilde{x}_{\sigma}^{T} x_{\sigma^{\prime}}=\delta_{\sigma \sigma^{\prime}}, \sum_{\sigma} x_{\sigma} \tilde{x}_{\sigma}^{T}=I$. $\mathbf{A}^{\sigma}(\mathbf{k})$ is the so-called Berry vector potential. Therefore the Berry curvature is obtained through the Stokes theorem as:

$$
\Omega_{k_{x} k_{y}}^{\sigma}=\frac{\partial}{\partial k_{x}} \mathbf{A}_{k_{y}}^{\sigma}-\frac{\partial}{\partial k_{y}} \mathbf{A}_{k_{x}}^{\sigma}=i \sum_{\sigma^{\prime} \neq \sigma} \frac{\tilde{x}_{\sigma}^{T} \frac{\partial H_{\mathrm{eff}}}{\partial k_{x}} x_{\sigma^{\prime}} \tilde{x}_{\sigma^{\prime}}^{T} \frac{\partial H_{\mathrm{eff}}}{\partial k_{y}} x_{\sigma}-\left(k_{x} \leftrightarrow k_{y}\right)}{\left(\omega_{\sigma}-\omega_{\sigma^{\prime}}\right)^{2}}
$$

Inserting the vector $x$ and the expression of matrix $H_{\text {eff }}$, we obtain

$$
\Omega_{k_{x} k_{y}}^{\sigma}=\sum_{\sigma^{\prime} \neq \sigma} \frac{i}{4 \omega_{\sigma} \omega_{\sigma^{\prime}}} \frac{\epsilon_{\sigma}^{\dagger} \frac{\partial D}{\partial k_{x}} \epsilon_{\sigma^{\prime}} \epsilon_{\sigma^{\prime}}^{\dagger} \frac{\partial D}{\partial k_{y}} \epsilon_{\sigma}-\left(k_{x} \leftrightarrow k_{y}\right)}{\left(\omega_{\sigma}-\omega_{\sigma^{\prime}}\right)^{2}}=\sum_{\sigma^{\prime} \neq \sigma} \Omega_{k_{x} k_{y}}^{\sigma \sigma^{\prime}}
$$

where $\Omega_{k_{x} k_{y}}^{\sigma \sigma^{\prime}}$ indicates the contribution to the Berry curvature of the band $\sigma$ from a different band $\sigma^{\prime}$. Therefore, the phonon Hall conductivity formula Eq. (S38) can be interpreted in terms of the Berry curvature.

\section{THE CALCULATION OF THE CHERN NUMBER}

The topological Chern number is obtained by integrating the Berry curvature over the first Brillouin zone as

$$
C^{\sigma}=\frac{1}{2 \pi} \int_{\mathbf{B Z}} d k_{x} d k_{y} \Omega_{k_{x} k_{y}}^{\sigma}
$$

For numerical calculation, we use

$$
C^{\sigma}=\frac{2 \pi}{L^{2}} \sum_{\mathbf{k}} \Omega_{k_{x} k_{y}}^{\sigma}
$$

where $\frac{1}{L^{2}} \sum_{\mathbf{k}}=\int \frac{d k_{x} d k_{y}}{(2 \pi)^{2}}$ and $V=L^{2} a, L^{2}$ is the area of the sample.

To calculate the integer Chern numbers, large $\mathbf{k}$-sampling points $N$ is needed. However there is always a zero eigenvalue at the $\Gamma$ point of the dispersion relation, which corresponds to a singularity of the Berry curvature. Therefore, we cannot sum up the Berry curvature very near this point to obtain Chern number of this band, unless we add a negligible on-site potential $\frac{1}{2} u^{T} V_{\text {onsite }} u$ to the original Hamiltonian. In Fig. S2(a), without the on-site potential, the Chern number of the fourth band is not an integer, no matter how large the sample size $N=N_{L}^{2}$ is $($ see Fig. S2 (b)). If we add the external on-site potential, the Chern number of the fourth band will become integer. In Fig. $\mathbf{S 2}$ (a), the $C_{4}$ changes gradually to -1 with increasing the on-site potential, while other Chern numbers do not change. And from Fig. S2(b), we see that with larger on-site potential, the Chern number of the fourth band could be an integer for smaller sample sizes.

\footnotetext{
$\dagger$ Electronic address: renjie@nus.edu.sg
}

* Electronic address: zhanglifa@nus.edu.sg 


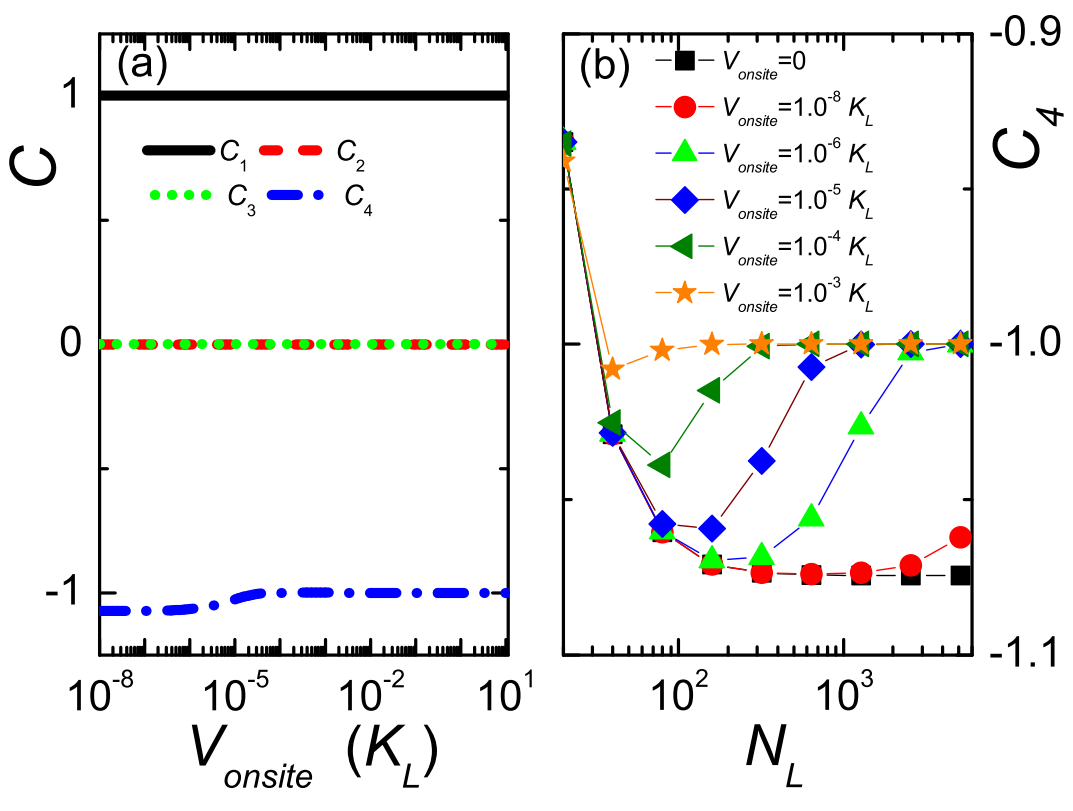

FIG. S2: (color online) (a) The four Chern number vs onsite potential $V_{\text {onsite. }}$ The unit for onsite potential is longitudinal spring constant $K_{L}$. Here $N=N_{L}^{2}=160000$; (b) The Chern number of the fourth band changes with $N_{L}$ for different onsite potentials. For both (a) and (b), $h=1 \mathrm{rad} / \mathrm{ps}$.

‡ Electronic address: phywjs@nus.edu.sg

$\S$ Electronic address: phylibw@nus.edu.sg

[1] A. Holz, Il Nuovo Cimento B 9, 83 (1972).

[2] L. Sheng, D. N. Sheng, and C. S. Ting, Phys. Rev. Lett. 96, 155901 (2006).

[3] Y. Kagan and L. A. Maksimov, Phys. Rev. Lett. 100, 145902 (2008).

[4] J.-S. Wang and L. Zhang, Phys. Rev. B. 80, 012301 (2009).

[5] L. Zhang, J.-S. Wang, and B. Li, New Journal of Physics 11, 113038 (2009).

[6] R. de L. Kronig, Physica (Amsterdam) 6, 33 (1939); J.
H. Van Vleck, Phys. Rev. 57, 426 (1940); R. Orbach, Proc. R. Soc. A 264, 458 (1961).

[7] Spin-Lattice Relaxation in Ionic Solids, edited by A. A. Manenkov and R. Orbach (Harper \& Row, New York, 1966).

[8] A. S. Ioselevich and H. Capellmann, Phys. Rev. B 51, 11446 (1995).

[9] R. J. Hardy, Phys. Rev. 132, 168 (1963).

[10] G. D. Mahan, Many-Particle Physics 3rd ed. (Kluwer Academic, New York, 2000).

[11] M. V. Berry, Proc. R. Soc. Lond. A 392, 45 (1984) 